\title{
Regulation of cytokine signaling pathways by PIAS proteins
}

\author{
Ke Shuai $^{1}$ \\ ${ }^{I}$ Division of Hematology-Oncology, Department of Medicine; Department of Biological Chemistry, University of California Los \\ Angeles, Los Angeles, California 90095, USA
}

Cytokines activate multiple signal transduction pathways to regulate gene expression. STATs and NF-kB are two important families of transcription factors activated by cytokines. Abnormal regulation of STAT and NF-kB activities has been associated with human diseases. The protein inhibitor of activated STAT (PIAS) protein family has been proposed to interact with over 60 proteins, many of which are transcription factors involved in the immune system. PIAS proteins regulate transcription through several mechanisms, including blocking the DNA-binding activity of transcription factors, recruiting transcriptional co-repressors and promoting protein sumoylation. This article is to review the role of PIAS proteins in the regulation of STAT and NF-kB signaling pathways.

Cell Research (2006) 16:196-202. doi:10.1038/sj.cr.7310027; published online 13 February 2006

Keywords: cytokine signaling, gene regulation, PIAS, STAT, NF-кB

\section{Introduction}

Cytokines are small polypeptides or glycoproteins secreted by a variety of cells to modulate cell growth and differentiation. Many cytokines are found to play important roles in regulating immune and inflammatory responses. Cytokines bind to their cell surface receptors to activate multiple signal transduction pathways to regulate gene expression. The distinct biological functions of cytokines largely result from the differential gene expression patterns triggered by cytokines. Thus, understanding the specificity of gene activation pathways is a fundamentally important question in the field of cytokine research.

Signal transducer and activator of transcription proteins (STATs) and nuclear factor- $\kappa \mathrm{B}(\mathrm{NF}-\kappa \mathrm{B})$ are two key families of transcription factors that are widely used by cytokines to modulate gene expression. Upon cytokine stimulation, STATs and NF- $\mathrm{KB}$ become activated in the cytoplasm, and then translocate into the nucleus where they activate transcription. These cytokine-mediated gene activation pathways are tightly controlled by both positive

Correspondence: Ke Shuai

Division of Hematology/Oncology, 11-934 Factor Bldg, 10833 Le Conte

Avenue, Los Angeles, California 90095-1678, USA

Tel: 310-206-9168; Fax: 310-825-2493;

E-mail:kshuai@mednet.ucla.edu and negative regulators. Abnormal cytokine signaling is associated with cancer and immune disorders. Biochemical and genetic studies have discovered a critical role of the PIAS (protein inhibitor of activated STAT) protein family in the regulation of cytokine signaling. This review focuses on how PIAS proteins regulate STAT and NF- $\mathrm{BB}$-mediated gene-activation pathways.

\section{The JAK-STAT signaling pathway}

The binding of a cytokine to its receptor leads to the receptor dimerization and the activation of JAK (Janus kinase) tyrosine kinases associated with the receptor. Specific tyrosine residues on the receptor are then phosphorylated by activated JAKs and serve as docking sites for recruiting a family of latent cytoplasmic transcription factors termed STATs (signal transducer and activator of transcription). STATs are phosphorylated by JAKs and subsequently dimerize and leave the receptor. The STAT dimers then translocate to the nucleus and direct immediate gene activation $[1,2]$. In addition to JAKs, STATs can also be activated by growth factor receptors and certain tyrosine kinases.

Seven members of the STAT family have been isolated: STAT1, STAT2, STAT3, STAT4, STAT5A, STAT5B, and STAT6. STATs are activated by many cytokines and growth factors, including IL-2, 3, 4, 5, 6, 7, 10, 12, GM-CSF, EPO, 
prolactin, growth hormone, EGF, PDGF and etc. For example, STAT1 is activated by interferon (IFN) stimulation and is required for IFN-mediated gene activation $[1,2]$. The physiological functions of STATs have been analyzed by genetic knockout studies. These studies demonstrate that different STATs have highly specific functions in mediating cytokine responses.

Several mechanisms to downregulate STAT signaling at various steps have been identified [3, 4]. 1) A family of cytokine-inducible proteins named SOCS (suppressor of cytokine signaling) can inhibit the JAK-STAT signaling through the inhibition of the tyrosine kinase activity of JAKs. 2) STAT signaling can be negatively regulated by protein tyrosine phosphatases (PTPase) that can inactivate JAKs and STATs. For example, the protein tyrosine phosphatase TC45 is responsible for the dephosphorylation of STAT1 in the nucleus[5]. 3) The transcriptional activity of STATs can be inhibited by a family of proteins named PIAS (protein inhibitor of activated STATs) [3, 6].

\section{The $\mathrm{NF}-\kappa \mathrm{B}$ signaling pathway}

$\mathrm{NF}-\kappa \mathrm{B}$ is a family of dimeric transcription factors composed of members of the Rel family of DNA-binding proteins $[7,8]$. NF- $\kappa \mathrm{B}$ can be activated by a large variety of signals, such as proinflammatory cytokins (TNF- $\alpha$, IL1), growth factors (PDGF), bacterial lipopolysaccharide (LPS), viruses (HIV-1, HTLV-1) and stress signals (UV, $\gamma$-irradiation, hypoxia). NF- $\kappa B$ is involved in mediating a wide spectrum of cellular responses, including infections, inflammation, as well as cell proliferation, transformation and apoptosis [9-11]. NF- $\kappa \mathrm{B}$ exerts its biological functions by transducing extracellular signals into the nucleus, where it can bind DNA and activate the transcription of specific genes. Inappropriate regulation of $\mathrm{NF}-\kappa \mathrm{B}$ is involved in a wide range of human diseases, including cancers, neurodegenerative disorders, ataxiatelangiectasia, arthritis, asthma, and other inflammatory conditions[12]. NF- $\mathrm{KB}$ is normally sequestered in the cytoplasm of nonstimulated cells by a group of inhibitory proteins, I $\mathrm{KBs}$, which bind $\mathrm{NF}-\mathrm{kB}$ and mask its nuclear localization sequence (NLS). Upon stimulation, I $\kappa$ Bs are rapidly phosphorylated by the upstream kinase IкB-kinase (IKK), which triggers the subsequent ubiquitination and proteolytic degradation of I $\kappa$ Bs. The free NF- $\kappa \mathrm{B}$ dimers can then translocate into the nucleus, bind to specific DNA sequences and regulate gene transcription.

Five mammalian NF- $\kappa \mathrm{B} /$ Rel proteins have been identi-

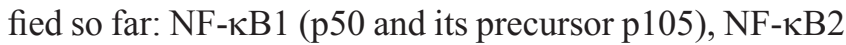
(p52 and its precursor p100), c-Rel, RelA (p65) and RelB[7, $8]$. All these proteins share a highly conserved 300 aa Rel homology region (RHR), which is responsible for dimer- ization, DNA-binding and interaction with the inhibitory I $\mathrm{B}$ proteins. It also contains a nuclear localization signal (NLS). The Rel proteins can form homo- or heterodimers between different subunits; however, they differ in their ability to activate transcription. Only RelA/p65 and c-Rel are found to contain transcriptional activation domains. It is believed that dimers composed solely of Rel proteins that lack transcriptional activation domains, such as p50, mediate gene repression.

NF- $\kappa \mathrm{B}$ activation induced by most stimuli is transient, indicating that the signal must be turned off. The downregulation of NF- $\kappa \mathrm{B}$ activity may occur at several levels. One is at the IKK inactivation, which appears to be caused by its autophosphorylation at the C-terminal regions of IKK $\alpha$ and IKK $\beta$ [13]. Hyperphosphorylation may result in a conformational change of the C-terminal region of IKK, which leads to a decrease in its kinase activity. Alternatively, it may make IKK more susceptible to a phosphatase, which inactivates IKK. IкB also plays an important role in terminating NF- $\kappa \mathrm{B}$ activation. I $\kappa \mathrm{B} \alpha$ itself is a downstream gene of NF- $\kappa B$ and serves as a negative feedback loop of $\mathrm{NF}-\kappa \mathrm{B}$ signaling. Newly synthesized IkBa induced by $\mathrm{NF}-\kappa \mathrm{B}$ activation enters the nucleus and binds to NF- $\kappa \mathrm{B}$, thereby enhancing its dissociation from DNA and causing its exportation to the cytoplasm through a nuclear export sequence (NES) present on IкB [14].

\section{Biochemical properties of PIAS proteins}

PIAS proteins were initially identified in studies aimed at the understanding of the JAK/STAT signaling pathway $[15,16]$. The mammalian PIAS family consists of PIAS1, PIAS3, PIASx and PIASy $[3,4,6,17]$. The existence of various PIAS isoforms adds to the complexity of the PIAS protein family. In Drosophila, a single PIAS homologue named dPIAS/Zimp has been identified [18, 19]. SIZ1 and SIZ2 are two PIAS-related proteins found in yeast [20].

\section{Domain structures of PIAS proteins}

The PIAS protein family contains several highly conserved regions (Figure 1). The most striking conserved domain of the PIAS family is a RING-finger-like zinc-bind-

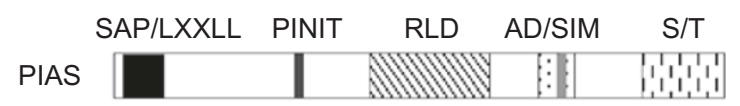

Figure 1 The domain structure of PIAS proteins. The conserved domains of PIAS proteins. SAP/LXXLL: SAF-A/B, Acinus, and PIAS domain. Within the SAP domain, there is a conserved LXXLL signature motif. RLD: RING finger-like zinc binding domain. AD/ SIM: acidic domain. SIM: SUMO1 Interaction Motif (SIM). S/T: serine-threonine rich region. 
ing domain (RLD). The SAF-A/B, Acinus and PIAS (SAP) domain is located at the $\mathrm{N}$-terminus of PIAS proteins, which is evolutionarily conserved in proteins ranging from yeast to human in origin and shared by other chromatin-binding proteins, such as scaffold attachment factor A and B (SAF$\mathrm{A}$ and $\mathrm{B}$ ) [21]. The SAP domain can recognize and bind to AT-rich DNA sequences present in scaffold-attachment regions/matrix-attachment regions (S/MAR) [22]. S/MAR elements, which are frequently found near gene enhancers, interact with nuclear matrix proteins to provide a unique nuclear microenvironment for transcriptional regulation. Within the SAP domain, a LXXLL signature motif is present in all PIAS proteins. The LXXLL signature motif has been shown to mediate interactions between nuclear receptors and their co-regulators [23]. The Pro-Ile-Asn-Ile-Thr (PINIT) motif represents a highly conserved region of PIAS proteins, which may be involved in the nuclear retention of PIAS3 [24]. A highly acidic region (AD) is also conserved in the PIAS family. Within the AD, a putative SUMO1 interaction motif (SIM) [25] is present in all PIASs except PIASy. The C-terminal regions of PIAS proteins are the least conserved. A Ser/Thr rich $(\mathrm{S} / \mathrm{T})$ region is found in all PIASs except PIASy.

\section{The SUMO ligase activity of PIAS}

Posttranslational modification of proteins by ubiquitin has been well characterized. The best known function for protein ubiquitination is to target protein for degradation [26]. A number of ubiquitin-like molecules have been identified, including SUMO (small ubiquitin-related modifier). Protein sumoylation has been suggested to regulate a wide variety of cellular processes, including targeting of proteins to the nucleus, protein-protein interactions, protein stability, formation of subnuclear structures, and modulation of transcription factors [27]

The mechanism for SUMO conjugation to target proteins is a pathway that is distinct from, but analogous to, ubiquitin conjugation, which involves three discrete steps: activation, conjugation, and ligation. The SUMO E3 ligase promotes the transfer of SUMO from E2 to the target substrate, forming an isopeptide bond between the carboxyl-terminus of SUMO and an $\varepsilon$-amino group of a Lys residue in the target protein $[28,29]$. Studies in yeast have identified two related proteins named SIZ1 and SIZ2 that possess E3 SUMO ligase activity [20]. SIZ1 can promote the sumoylation of yeast septins in vitro. The removal of SIZ1 and SIZ2 almost completely abolished the overall protein sumoylation in yeast. SIZ1 and SIZ2 are homologous to the mammalian PIAS proteins. Subsequently, it has been shown that members of the PIAS family can participate in the sumoylation of a number of proteins, including $\mathrm{p} 53$, androgen receptor (AR), and SMAD $[3,6]$.

\section{Mechanisms of PIAS-mediated gene regulation}

PIAS proteins have now been suggested to regulate the activity of over 60 proteins [6]. Several molecular mechanisms have been proposed to explain how PIAS proteins might regulate transcription (Figure 2). 1) PIAS may inhibit transcription by blocking the DNA-binding activity of a transcription factor; 2) PIAS may repress transcription by recruiting other co-regulators, such as histone deacetylases (HDACs); 3) PIAS may regulate transcription by promoting the sumoylation of a transcription factor. It has been shown that sumoylation can either positively or negatively influence the activity of a transcription factor; 4) PIAS may repress transcription by sequestering transcription factors to certain subnuclear structures where co-repressor complexes

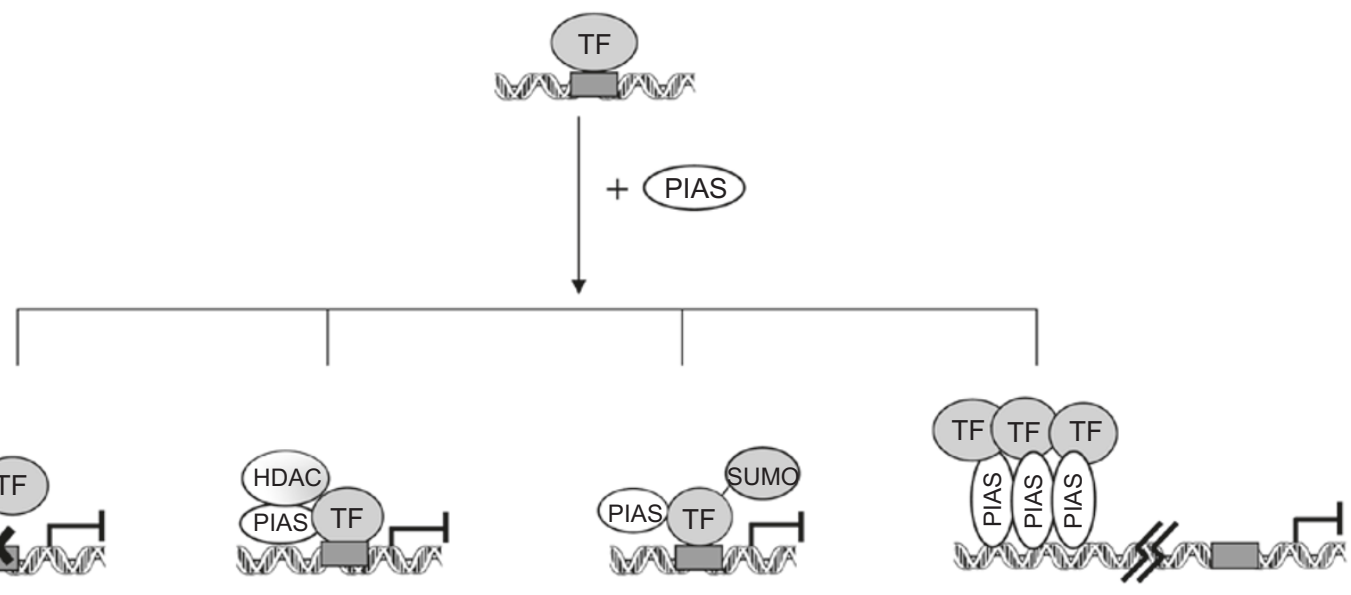

Figure 2 Proposed models of PIAS-mediated transcriptional regulation. Four possible mechanisms have been proposed to account for PIAS-mediated transcriptional regulation. See text for details. 


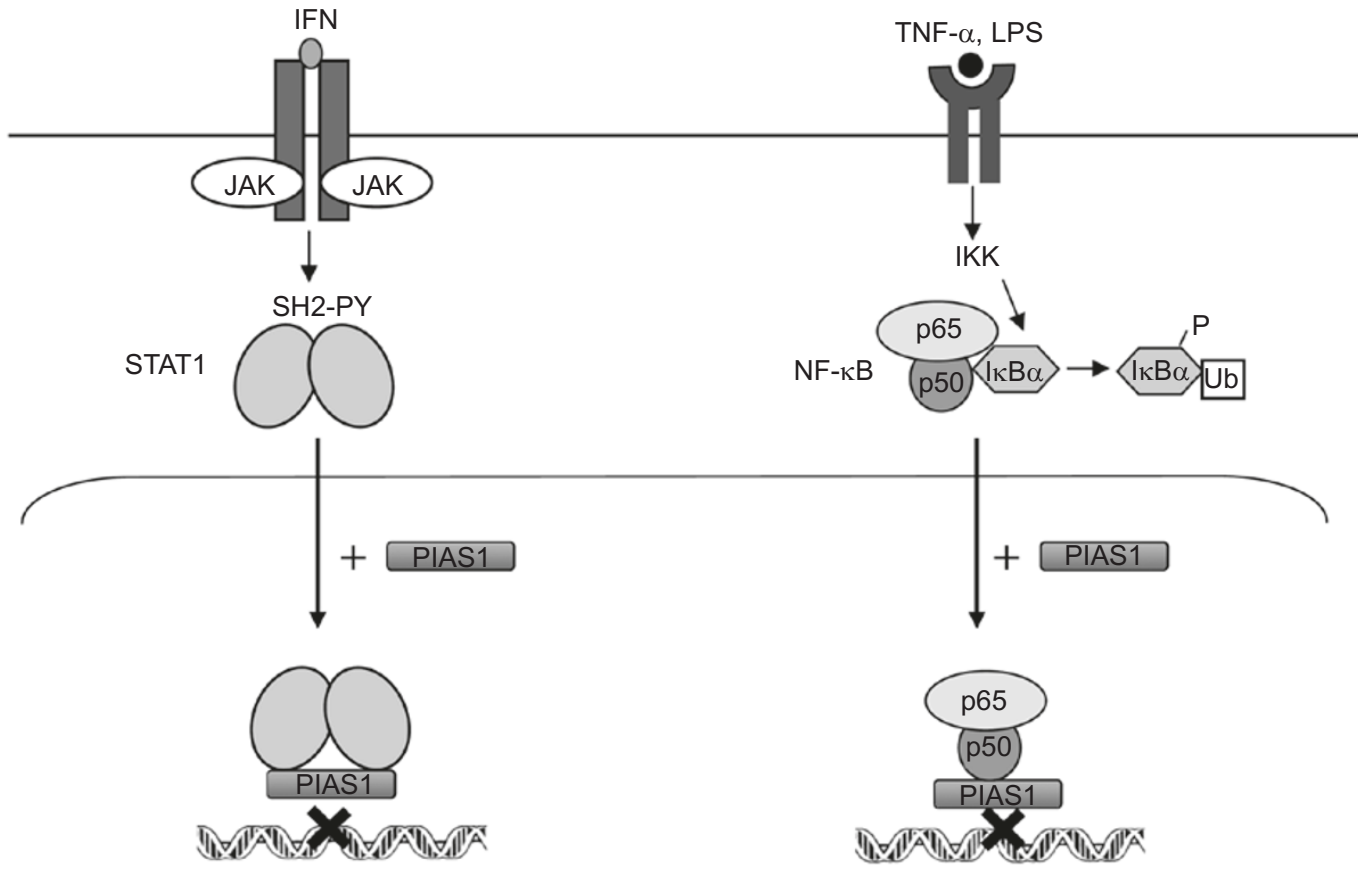

Figure 3 PIAS1 regulates both STAT and NF- $\kappa$ B signaling pathway. PIAS1 selectively inhibits a subset of STAT1- or NF- $\kappa$ B-dependent genes in response to IFN or TNF (or LPS) treatment, respectively [31,36].

are enriched. Although endogenous PIAS1 and PIASy proteins were found to be expressed uniformly in the nucleus $[30,31]$, it remains possible that PIAS proteins may form nuclear dots when challenged with certain stimuli.

\section{PIAS proteins in the regulation of cytokine signaling}

Numerous studies have shown that PIAS proteins play an important role in the regulation of the STAT and NF- $\kappa B$ signaling pathways (Figure 3 ).

\section{PIAS in the regulation of STAT signaling}

The interaction of PIAS with a STAT molecule was initially revealed by yeast two-hybrid assays $[15,16]$. Subsequently, members of the PIAS family have been shown to interact with various STATs. The endogenous STAT-PIAS interaction has been analyzed by in vivo coimmunoprecipitation assays using specific anti-PIAS antibodies. There exists specificity as well as redundancy in PIAS-STAT interactions. PIAS1, PIAS3 and PIASx interact with STAT1, STAT3 and STAT4, respectively $[15,16,32]$, and PIASy also interacts with STAT1 [30]. The interaction of PIAS and STAT requires cytokine stimulation. It has been suggested that PIAS interacts with the dimeric form, but not the monomeric form of STAT, which may explain the requirement of cytokine stimulation for PIAS-STAT association [33].

The binding of PIAS to STAT results in the inhibition of STAT-mediated gene activation. PIAS1 and PIAS3 inhibit STAT-mediated gene activation by blocking the DNA binding activity of STAT $[15,16]$. By contrast, PIASy and PIASx repress STAT1 and STAT4-medaited gene activation without affecting STAT DNA binding activity. PIASy and PIASx act by recruiting other co-repressor molecules such as HDACs to inhibit transcription [30, 32].

It has been shown that STAT1 can be sumoylated on Lys 703, which can be strongly enhanced by PIAS proteins $[34,35]$. In addition, IFN- $\gamma$ treatment appears to induce STAT1 sumoylation [35]. However, whether the SUMO E3 ligase activity of PIAS1 is involved in regulating STAT1 signaling is highly controversial. While Rogers et al, showed that the mutation of Lys703 to Arg did not affect the activation of STAT1 or the ability of PIAS1 to repress STAT1-mediated gene activation[34], Ungureanu et al, showed that the same mutation resulted in increased STAT1-dependent gene activation in response to IFN- $\gamma$ [35]. It is possible that the effect of sumoylation on the activity of STAT may be dependent on the promoter context of individual genes. Clearly, the physiological importance of PIAS SUMO-ligase activity in gene regulation remains to be clarified and further studies are needed to understand how protein SUMO modification can regulate the activity of STAT in vivo.

Genetic studies have been performed to examine the 
physiological role of PIAS proteins in gene regulation. In Drosophila, the removal of dPIAS resulted in hyperactive JAK-STAT signaling and increased blood-tumor formation [19]. Gene-targeting studies in mice have revealed a critical role of PIAS in the proper regulation of cytokine signaling. Pias $1^{-/}$mice are produced at a frequency $50 \%$ lower than the expected Mendelian ratio due to partial perinatal lethality. The surviving PIAS1-deficient mice are runted compared with their wild-type littermates [36]. Wild type and Pias $^{-/-}$bone-marrow-derived macrophages (BMDMs) and primary mouse embryonic fibroblasts (MEFs) have been used to analyze the physiological role of PIAS1 in STAT1 signaling [36]. Quantitative PCR analysis indicates that the removal of PIAS1 leads to the increased transcriptional activation of STAT1-dependent genes in response to IFN- $\beta$ or IFN- $\gamma$ stimulation. Most interestingly, gene expression profiling studies showed that PIAS1-deficiency affects the expression of only a subset of IFN-stimulated genes (PIAS1-sensitive genes). For example, the transcription of $G B P 1$ (guanylate binding protein), $M I G$ (monokine induced by IFN-gamma) and IPIO (IFN-gamma-inducible protein $10 \mathrm{kDa}$ ) in responding to IFN- $\gamma$ was enhanced by 2-5 fold in Pias 1 null cells as compared to that in wild type cells, while the transcription of $I R F 1$ (interferon regulatory factor-1), NOS2 (nitric-oxide synthase 2) or SOCS1 was not altered [36]. Thus, unlike other general negative regulators of JAK-STAT signaling pathways, PIAS1 does not inhibit the entire IFN-responsive genes. This unexpected specificity of PIAS1 in the regulation of STAT1-mediated gene activation suggests that cytokine-activated genes can be negatively regulated in subgroups by inhibitory molecules such as PIAS1.

To understand the molecular basis of PIAS1 specificity in STAT 1 gene activation, chromatin immunoprecipitation (ChIP) assays were performed using wild type and Pias 1 null cells. Interestingly, an increased binding of STAT1 to the promoters of PIAS1-sensitive genes, but not PIAS1insensitive genes, in PIAS1-deficient macrophages was observed [36]. These results suggest that the specificity of PIAS1 on STAT1-mediated gene regulation may be determined, at least in part, by the promoter microenvironment of STAT1-target genes. In addition, it was found that the promoters of PIAS1-sensitive genes contain weaker STAT1 binding sites than those of PIAS1-insensitive genes. It is possible that STAT1 binding sites with lower affinity are more sensitive to the level of PIAS1 expression [36].

Although PIAS1 affects only a subset of IFN-responsive genes, PIAS1 plays critical roles in innate immunity. The antiviral activity of IFN- $\gamma$ or IFN- $\beta$ was significantly increased in Pias $1^{-/}$cells [36]. In addition, Pias $1^{-/-}$mice displayed increased protection against bacterial infection and showed enhanced antiviral response against vesicular stomatitis virus (VSV) infection [36]. These results are consistent with a negative regulator role of PIAS1 in IFNtriggered STAT1 signaling.

The generation of Piasy ${ }^{-/-}$mice has been reported by two independent groups which showed that Piasy ${ }^{-1-}$ mice had no obvious defects in animal development [37, 38]. It will be interesting to examine if the lack of an obvious phenotype of Piasy ${ }^{-/}$mice may result from a functional redundancy in PIAS proteins.

\section{PIAS in the regulation of $N F-\kappa B$ signaling}

Since STAT1 and the Rel family of proteins share high structural similarity in their DNA binding domains [39], biochemical studies had been performed to explore the possibility of PIAS1 in the regulation of the NF- $\mathrm{kB}$ pathway. Both PIAS1 and PIAS3 can interact with NF-кB p65 $[31,40]$. In NF- $\kappa$ B reporter assays, PIAS1 and PIAS3 can repress the transcriptional activity of p65 [31, 40]. In addition, the induction of several endogenous NF- $\kappa B$-target genes, such as those encoding $I \kappa B \alpha$ and $B f l-1$, in response to TNF stimulation was inhibited in PIAS1-overexpression cells [31]. Similar to the case of STAT1, purified PIAS1 inhibited the DNA binding activity of NF- $\kappa$ B in mobility gel-shift assays. In addition, PIAS1 repressed the transcriptional activity of a GAL4-p65 fusion protein when assayed using the $\kappa \mathrm{B}$ reporter, but not the GAL4 reporter. Consistently, ChIP assays showed that the recruitment of p65 to the endogenous promoter of $I \kappa B \alpha$, a known NF- $\kappa \mathrm{B}$-target gene, was inhibited in PIAS1-overexpressing cells, but was enhanced in PIAS1-deficient cells [31].

The physiological role of PIAS 1 in the regulation of NF$\mathrm{kB}$ was examined using Pias 1 null mice. The transcription of $I \kappa B a, I L-1 \beta$ and JunB in response to TNF- $\alpha$ treatment was enhanced in Pias 1 null cells as compared to that in wild type controls, while the induction of other NF-kB target genes, including Cxcl10 (CXC-chemokine ligand 10), Nos2, and Mcp1 (Monocyte chemotactic protein-1), was not significantly affected in the absence of PIAS1[31]. Detailed microarray studies showed that PIAS1 affects approximately $48 \%$ of TNF-induced genes. These data indicate, similar to the case of PIAS1 in STAT signaling, PIAS1 displays specificity by regulating only a subset of $\mathrm{NF}-\kappa \mathrm{B}$-mediated genes in vivo.

Consistent with gene expression analysis in Pias1 null cells, serum levels of IL- $1 \beta$ and TNF- $\alpha$ were elevated by 6-fold and 3.5-fold, respectively, in Pias1 null mice as compared to their wild type littermates[31]. In contrast, serum levels of IFN- $\gamma$ and IL-4, two cytokines not directly regulated by the NF- $\kappa \mathrm{B}$ pathway, were not significantly altered in Pias 1 null mice. Furthermore, Pias $1^{-/-}$mice were hypersensitive to LPS-induced endotoxic shock [31]. These 
data indicate that PIAS1 is a physiologically important negative regulator of $\mathrm{NF}-\kappa \mathrm{B}$ signaling.

\section{Conclusions and Perspectives}

Since the original discovery of a role of PIAS proteins in STAT signaling, PIAS proteins have now been reported to be involved in the regulation of over 60 proteins, many of which are transcription factors involved in the immune system. Recent genetic studies using Pias 1 knockout mice have validated a critical role of PIAS1 in the regulation of STAT1 and NF- $\kappa B$ signaling under physiological settings (Figure 3). The unexpected findings that PIAS1 displays specificity in cytokine signaling by regulating only a subgroup of genes strongly support a hypothesis that cytokine-responsive genes can be selectively downregulated by specific negative regulators such as PIAS1. Targeting gene-specific regulators may represent a novel cytokine-based therapeutic strategy. Since STAT and NF$\mathrm{kB}$ play important roles in immunity and cancer, it will be interesting to explore if dysregulation of PIAS proteins is associated with cancer and immune disorders.

Although PIAS proteins possess SUMO E3 ligase activity, the physiological role of PIAS-mediated protein sumoylation in the regulation of cytokine signaling pathways remains to be determined. One challenge is to identify and characterize physiological substrates of PIAS SUMO ligases. Another important area of PIAS research is to understand how the activity of PIAS proteins is regulated by cytokines. Further studies on PIAS proteins will significantly enhance our understanding of the regulation of cytokine signaling pathways.

\section{Acknowledgements}

Supported by grants from The National Institutes of Health, USA (Kei Shuai).

\section{References}

1 Darnell JE Jr. STATs and gene regulation. Science 1997; 277:1630-5.

2 Levy DE, Darnell JE. Stats: transcriptional control and biological impact. Nat Rev Mol Cell Biol 2002; 3:651-62.

3 Shuai K, Liu B. Regulation of JAK-STAT signaling in the immune system. Nat Rev Immunol 2003; 3:900-11.

4 Shuai K. Modulation of STAT signaling by STAT-interacting proteins. Oncogene 2000; 19:2638-44.

5 ten Hoeve J, de Jesus Ibarra-Sanchez M, Fu Y, et al. Identification of a nuclear Stat1 protein tyrosine phosphatase. Mol Cell Biol 2002; 22:5662-8.

6 Shuai K, Liu B. Regulation of gene-activation pathways by PIAS proteins in the immune system. Nat Rev Immuno 2005; 5:593605.
7 Karin M, Ben-Neriah Y. Phosphorylation meets ubiquitination: the control of NF-[kappa]B activity. Annu Rev Immunol 2000; 18:621-63.

8 May MJ, Ghosh S. Rel/NF-kappa B and I kappa B proteins: an overview. Semin Cancer Biol 1997; 8:63-73.

9 Viatour P, Merville MP, Bours V, Chariot A. Phosphorylation of NF-kappaB and IkappaB proteins: implications in cancer and inflammation. Trends Biochem Sci 2005; 30:43-52.

10 Bonizzi G, Karin M, Yamamoto Y, Wang QM. The two NF-kap$\mathrm{paB}$ activation pathways and their role in innate and adaptive immunity. Trends Immunol 2004; 25:280-8.

11 Karin M, Yamamoto Y, Wang QM. The IKK NF-kappa B system: a treasure trove for drug development. Nat Rev Drug Discov 2004; 3:17-26.

12 Aggarwal BB. Signaling pathways of the TNF superfamily: a double-edged sword. Nat Rev Immunol 2003; 3:745-56.

13 Delhase M, Hayakawa M, Chen Y, Karin M. Positive and negative regulation of IkappaB kinase activity through IKKbeta subunit phosphorylation. Science 1999; 284:309-13.

14 Arenzana-Seisdedos F, Turpin P, Rodriguez M, et al. Nuclear localization of I kappa B alpha promotes active transport of NF-kappa B from the nucleus to the cytoplasm. J Cell Sci 1997; 110:369-78.

15 Chung CD, Liao J, Liu B, et al. Specific inhibition of Stat 3 signal transduction by PIAS3. Science 1997; 278:1803-5.

16 Liu B, Liao J, Rao X, et al. Inhibition of Stat1-mediated gene activation by PIAS1. Proc Natl Acad Sci U S A 1998; 95:10626-31.

17 Shuai K. The STAT family of proteins in cytokine signaling. Prog Biophy Mol Bio 1999; 71:405-22.

18 Mohr SE, Boswell RE. Zimp encodes a homologue of mouse Miz1 and PIAS3 and is an essential gene in Drosophila melanogaster. Gene 1999; 229:109-16.

19 Betz A, Lampen N, Martinek S, Young MW, Darnell JE, Jr.. A Drosophila PIAS homologue negatively regulates stat92E. Proc Natl Acad Sci U S A 2001; 98:9563-8.

20 Johnson ES, Gupta AA. An E3-like factor that promotes SUMO conjugation to the yeast septins. Cell 2001; 106:735-44.

21 Aravind L, Koonin EV. SAP - a putative DNA-binding motif involved in chromosomal organization. Trends Biochem Sci 2000; 25:112-4.

22 Kipp M, Gohring F, Ostendorp T, et al. SAF-Box, a conserved protein domain that specifically recognizes scaffold attachment region DNA. Mol Cell Biol 2000; 20:7480-9.

23 Glass CK, Rosenfeld MG. The coregulator exchange in transcriptional functions of nuclear receptors. Genes and Development 2000; 14:121-41.

24 Duval D, Duval G, Kedinger C, Poch O, Boeuf H. The 'PINIT' motif, of a newly identified conserved domain of the PIAS protein family, is essential for nuclear retention of PIAS3L. FEBS Lett 2003; 554:111-8.

25 Minty A, Dumont X, Kaghad M, Caput D. Covalent modification of p73alpha by SUMO-1. Two-hybrid screening with p73 identifies novel SUMO-1-interacting proteins and a SUMO-1 interaction motif. J Biol Chem 2000; 275:36316-23.

26 Pickart CM. Mechanisms underlying ubiquitination. Annu Rev Biochem 2001; 70:503-33.

27 Johnson ES. Protein modification by SUMO. Annu Rev Biochem 2004; 73:355-82.

28 Kim KI, Baek SH, Chung $\mathrm{CH}$. Versatile protein tag, SUMO: 
its enzymology and biological function. J Cell Physiol 2002; 191:257-68.

29 Melchior F. SUMO — nonclassical ubiquitin. Annu Rev Cell Dev Biol 2000; 16:591-626.

30 Liu B, Gross M, ten Hoeve J, Shuai K. A transcriptional corepressor of Stat 1 with an essential LXXLL signature motif. Proc Natl Acad Sci U S A. 2001; 98:3203-7.

31 Liu B, Yang R, Wong KA, et al. Negative regulation of NF-kappaB signaling by PIAS1. Mol Cell Biol 2005; 25:1113-23.

32 Arora T, Liu B, He H, et al. PIASx Is a transcriptional co-repressor of signal transducer and activator of transcription 4. J Biol Chem 2003; 278:21327-30.

33 Liao J, Fu Y, Shuai K. Distinct roles of the NH2- and COOHterminal domains of the protein inhibitor of activated signal transducer and activator of transcription (STAT)1 (PIAS1) in cytokine-induced PIAS1-Stat1 interaction. Proc Natl Acad Sci U S A 2000; 97:5267-72.

34 Rogers RS, Horvath CM, Matunis MJ. SUMO modification of STAT1 and its role in PIAS-mediated inhibition of gene activa- tion. J Biol Chem 2003; 278:30091-7.

35 Ungureanu D, Vanhatupa S, Kotaja N, et al. PIAS proteins promote SUMO-1 conjugation to STAT1. Blood 2003; 102:3311-3.

36 Liu B, Mink S, Wong KA, et al. PIAS1 selectively inhibits interferon-inducible genes and is important in innate immunity. Nat Immunol 2004; 5:891-8.

37 Wong KA, Kim R, Christofk H, Gao J, Lawson G, Wu H. Protein inhibitor of activated STAT Y (PIASy) and a splice variant lacking exon 6 enhance sumoylation but are not essential for embryogenesis and adult life. Mol Cell Biol 2004;24:5577-86.

38 Roth W, Sustmann C, Kieslinger M, et al. PIASy-deficient mice display modest defects in IFN and Wnt signaling. J Immunol 2004; 173:6189-99.

39 Chen X, Vinkemeier U, Zhao Y, Jeruzalmi D, Darnell JE, Jr., Kuriyan J. Crystal structure of a tyrosine phosphorylated STAT-1 dimer bound to DNA. Cell 1998; 93:827-39.

40 Jang HD, Yoon K, Shin YJ, Kim J, Lee SY. PIAS3 suppresses NF-kappaB-mediated transcription by interacting with the p65/ RelA subunit. J Biol Chem 2004; 279:24873-80. 